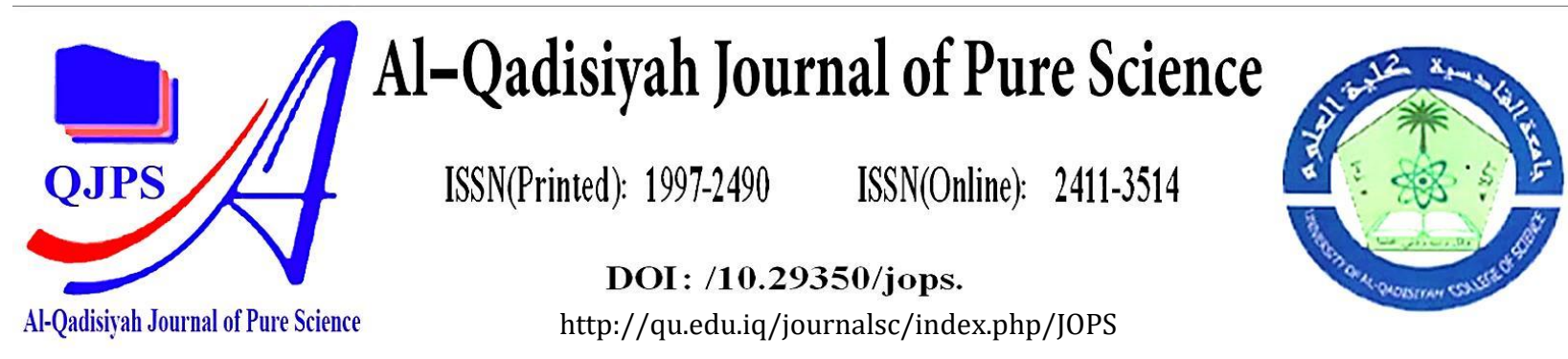

\title{
Study of some histopathological changes occurring in white laboratory mice infected with Cutaneous Leishmaniasis in Al - diwaniyah province, Iraq.
}

\begin{tabular}{|c|c|}
\hline \multirow{2}{*}{$\begin{array}{l}\text { Authors Names } \\
\text { a. Lubna.A.Al-ibrahimi } \\
\text { b. Hind Al-shabani } \\
\text { c. Marwa sami alwan }\end{array}$} & ABSTRACT \\
\hline & Leishmaniasis is caused by an intracellular parasite. It is endemic in \\
\hline Article History & 2 million new cases occur each year. The present study aims to follow up \\
\hline Received on: $3 / 6 / 2021$ & infected mice with Leishmania.major .The histological study of the liver \\
\hline $\begin{array}{l}\text { Revised on: } 1 / 7 / 2021 \\
\text { Accepted on: } 11 / 7 / 2021\end{array}$ & tissue of white laboratory mice (Mus musculus) infected with L. major a \\
\hline Keywords: & Hepatocyte degeneration, And loss of the radial arrangement of \\
\hline $\begin{array}{l}\text { Leishmaniasis. Leishmania. } \\
\text { histopathological changes } \\
\text { mice }\end{array}$ & $\begin{array}{l}\text { hepatocytes, With heavy infiltration in inflammatory cells, especially } \\
\text { phagocytes( Macrophage) with Hyperplasia and congestion of the bile }\end{array}$ \\
\hline $\begin{array}{l}\text { DOI: https://doi.org/10.29350/ } \\
\text { jops. 2021.26. } 4.1323\end{array}$ & $\begin{array}{l}\text { 'were showed epidermal ulcerative , Accompanied by severe leaching of } \\
\text { the dermis layer neutrophil ,polymorph lymphocytes 'with hemorrhage of } \\
\text { the dermis, with necrosis of the epidermal cells of all skin lesions in the } \\
\text { ear, foot and tail. }\end{array}$ \\
\hline
\end{tabular}

\section{Introduction}

Leishmaniasis one of the important diseases for humans, which is caused by the parasite Leishmania Which belongs to the order Kinetoplastida family Trypanosomatid. Leishmania parasite two forms during its life cycle Promastigote form It is an infectious stage and is seen in females of the sand fly Phlebotomus (Carrier host) in the ancient world .And Lutzomyia In the new world $(18,19)$. As well as in industrial agricultural communities. Amastigote form It is found in humans and storing hosts and lives inside macrophages in the skin, mucous membranes, lymph nodes, bone marrow and spleen.(10,4). Leishmaniasis is characterized by a variety of clinical features of the disease from Cutaneous Leishmaniasis (CL) Mucocutaneous Leishmaniasis (MCL) Visceral Leishmaniasis (VL) $(6,12)$. Cutaneous leishmaniasis appears in two types, the first Zoonotic Cutaneous Leishmaniasis (ZCL) It is caused by a parasite L. major And it appears in rural areas and causes a wet sore . and Anthroponotic Cutaneous Leishmaniasis (ACL) When the parasite is transmitted from one person to another and causes it L. tropica This type causes a dry sore $(7,9)$ 
Leishmaniasis represents a major public health problem in the Eastern Mediterranean Region (EMR) of the World Health Organization (WHO) (13), leishmaniasis causes 70,000 deaths per year. Clinical features depend on the species of Leishmania involved and the immune response of the host .Infection of mice with L. major is a well-established model for the investigation of factors that control disease development. Most inbred strains of mice, termed resistant or healer strains, develop a local inflammation at the site of L. major inoculation (17).

\section{Material and Methods}

\section{Samples Collection}

Leishmaniasis samples were collected from the edge of ulcers before treatment for patients with cutaneous leishmaniasis, and arrivals to Diwaniyah Teaching Hospital.

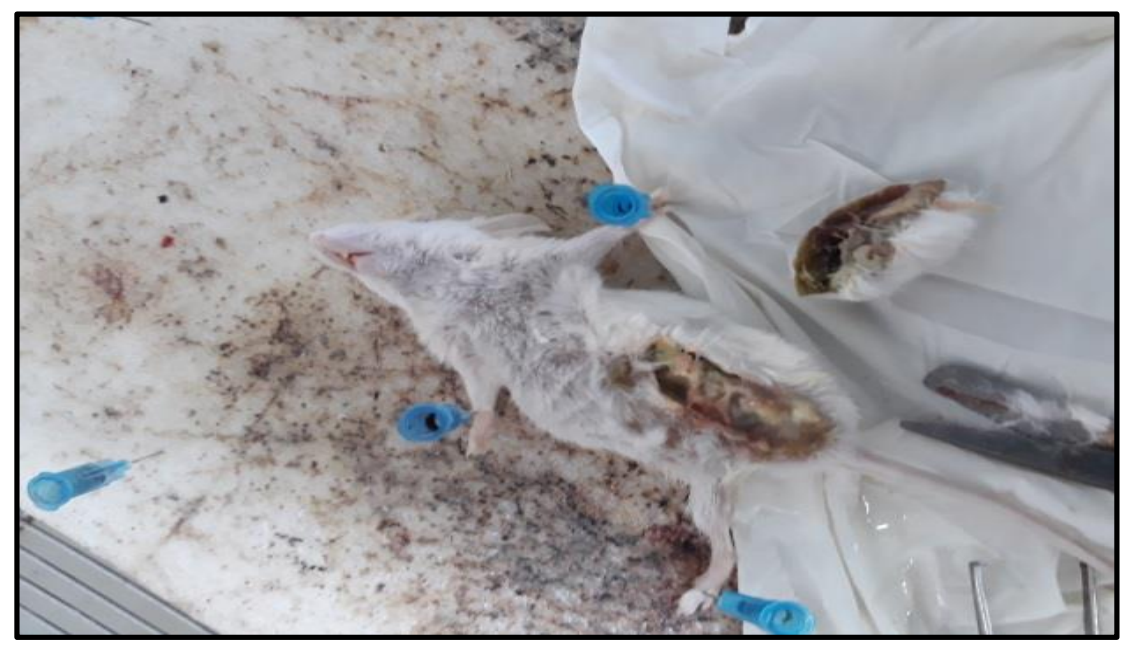

Fig (1): Represents some of the skin lesions caused by infection with leishmaniasis dermal parasites.

\section{Diagnosis of Samples}

Clinical diagnosis : Clinical diagnosis was made by a dermatologist.

Laboratory diagnosis: The direct smear method prepared from the edge of a pigmented ulcer using Giemsa was used and examined by a high-strength microscope using an oil immersion.(8)

\section{Results}

\section{1- Gross Changes}

The current study showed the emergence of skin infections in mice experimentally infected with Leishmania parasite, which were observed visually in the injection areas a month after the infection. These lesions were accompanied by changes in the behavior of the affected animals, such as lack of movement and lack of desire for food in addition to lethargy.

\section{2- Histological Changes}

The first group (control group) showed that normal liver tissue consisted of hepatocytes arranged radially in hexagonal shapes, with the bile duct appearing normal, Fig (2). 


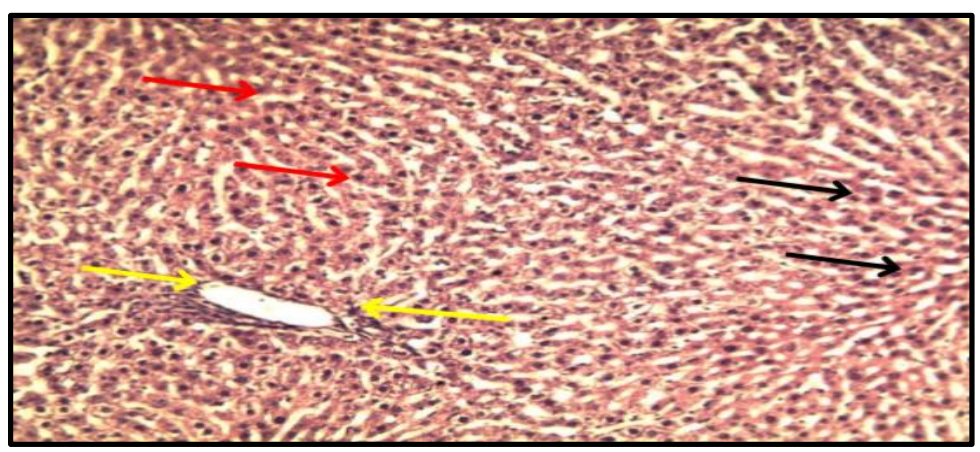

Fig (2): A cross section of mouse liver tissue from the control group shows the normal tissue of the liver. With the appearance of the bile duct normal (yellow arrow) (. (H \& E, 10X)

The results of the study show that the histological sections of the liver of laboratory mice infected with L. major, hepatocytes are irregular in shape as a result of severe fatty degeneration of hepatocytes, which creates vacuoles (expansion of hepatic sinuses) leading to the loss of the radial arrangement of the hepatocytes, with the appearance of heavy infiltration Inflammatory cells, especially macrophages, with Hyperplasia and congestion of the bile duct.fig $(3,4)$

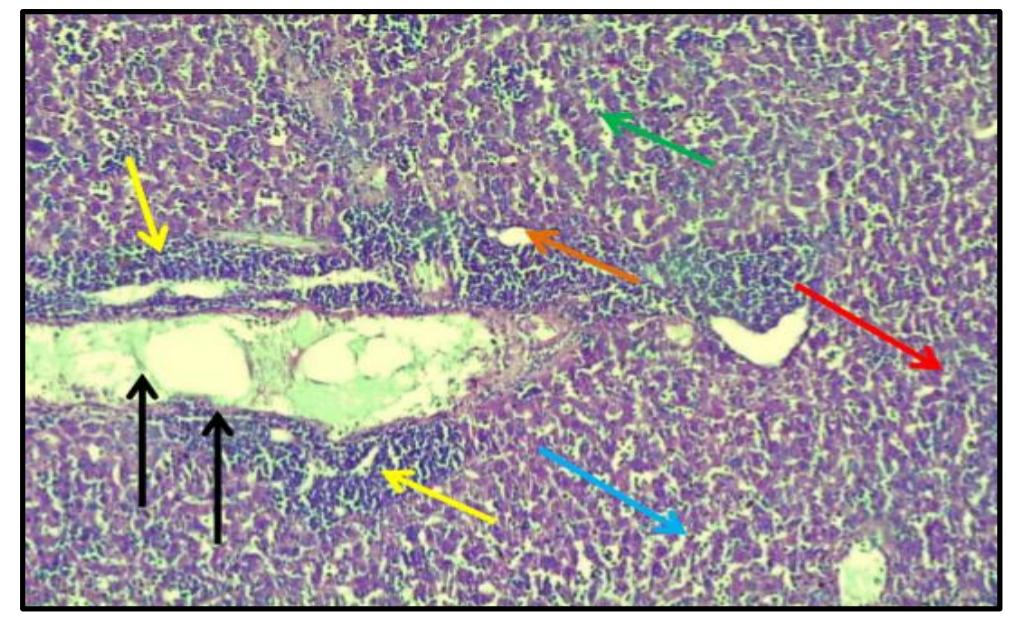

Fig (3): A cross section of the liver tissue of the affected experiment group observes the disappearance of the radial arrangement between the hepatocytes (green arrow) and the infiltration of inflammatory cells, especially phagocytes (large macrophage) (yellow arrow) with severe fatty degeneration, the nucleus takes a peripheral position (red and blue arrow) ). Hepatic sinus enlargement (orange arrow) and biliary enlargement and congestion (black arrow) (H\&E, 10x). 


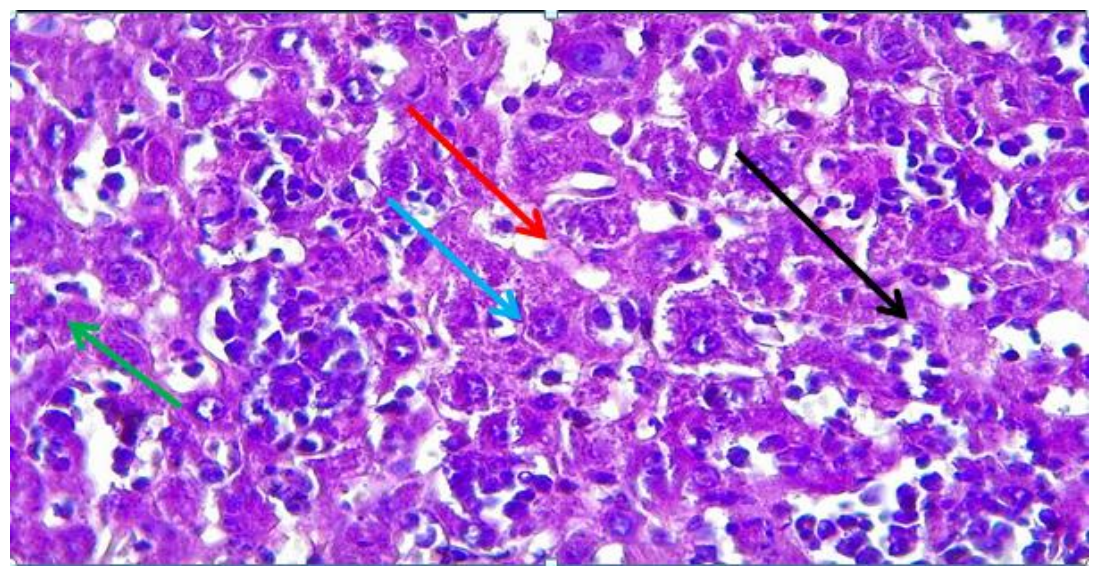

Fig (4) a cross-section in the liver tissue of the affected experiment group. The radial arrangement between the hepatocytes (green arrow) and the infiltration of inflammatory cells, especially phagocytic cells (large macrophage) (yellow arrow) with severe steatosis, the nucleus takes a peripheral position (red and blue arrow). Hepatic sinus enlargement (orange arrow) and bile duct enlargement and congestion (black arrow) (H \& E, 40X).

The study of histological sections of skin lesions taken from the ear, foot and tail revealed epidermal ulcerative ulcers accompanied by severe infiltration in the dermis layer mediated by neutrophil cells and polymorphic lymphocytes, with the appearance of severe hemorrhage in the area of the dermis layer, with necrosis in the Epidermal cells for all skin lesions in the ear, foot and tail, Fig $(5,6,7)$.

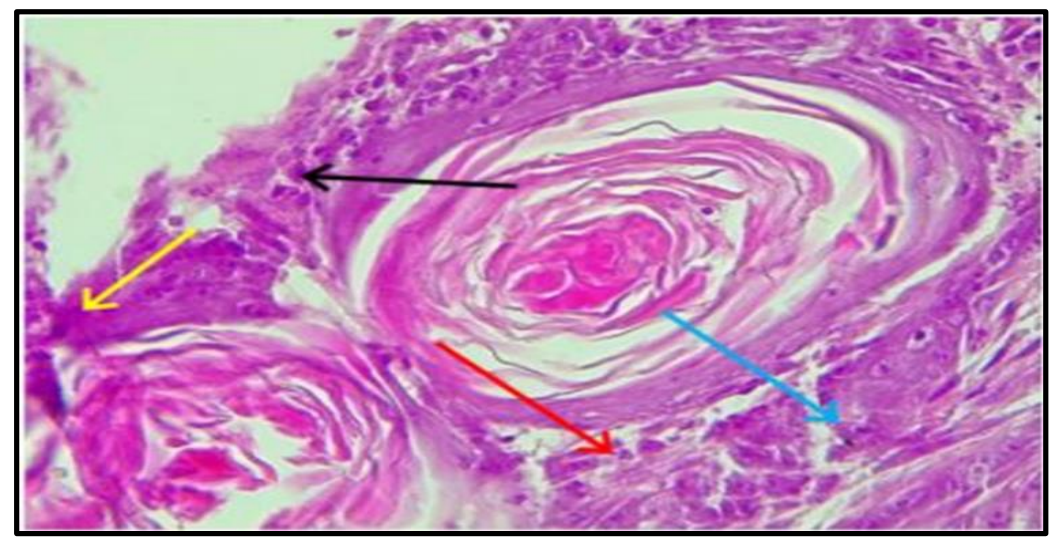

Fig (5) A cross-section in the ear of the affected experimental group, in which skin ulcers are observed with severe inflammatory infiltration of the dermis layer by neutrophils and polymorphic lymphocytes (red and blue arrow). With severe hemorrhage in the dermis (yellow arrow). Necrosis of the epidermal cells in the ear (black arrow). (H\&E,40X) . 


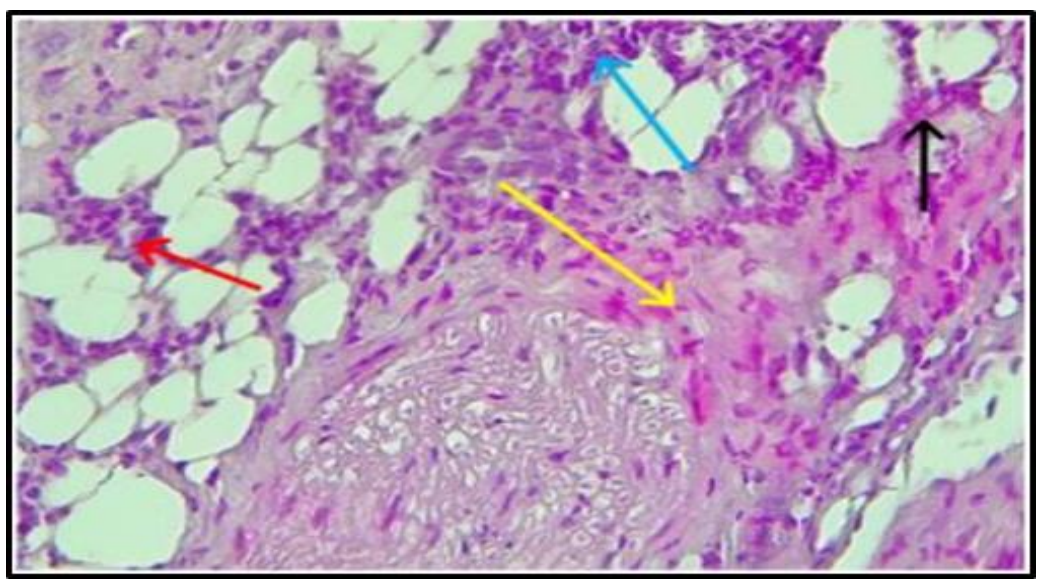

Fig (6) A cross section of the foot of the affected experimental group, in which skin ulcers are observed with severe inflammatory infiltration of the dermis layer by neutrophils and polymorphic lymphocytes (red and blue arrow). With severe hemorrhage in the dermis (yellow arrow). Necrosis of the epidermal cells in the ear (black arrow) (H\&E, 400X) .

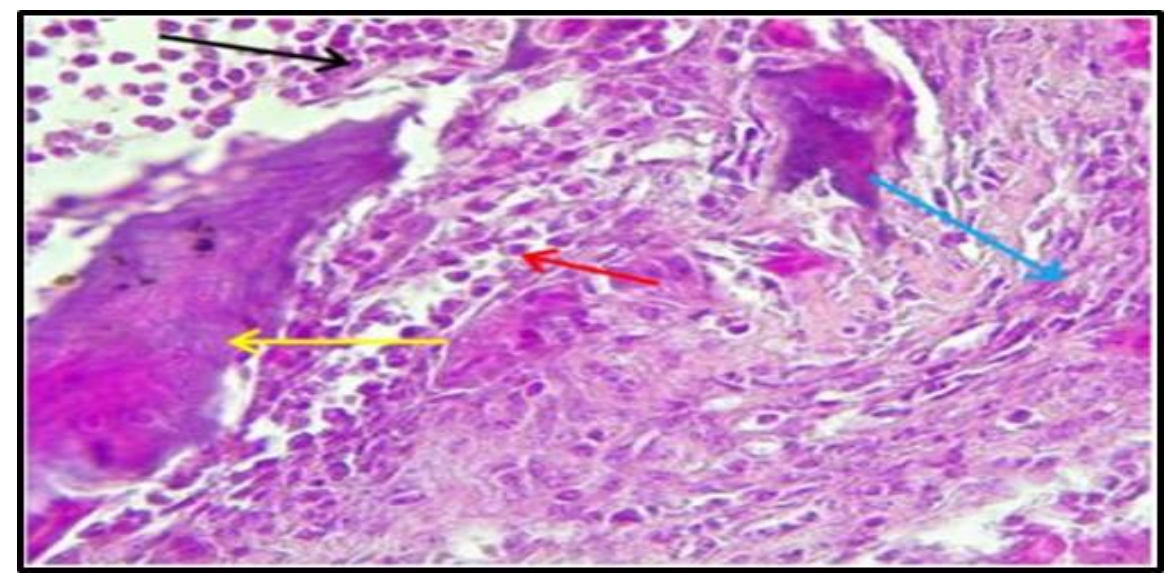

Fig (7) A cross section in the tail of the affected experimental group, in which skin ulcers are observed with severe inflammatory infiltration of the dermis layer by neutrophils and polymorphic lymphocytes (red and blue arrow). With severe hemorrhage in the dermis (yellow arrow). Necrosis of the epidermal cells in the ear (black arrow). (H\&E,40X).

\section{Discussion}

The results of histopathology of the livers of infected mice showed that the cutaneous leishmaniasis parasite is widespread in the liver and organs close to the surface of the skin, although it appears in the dermal region. In the study of (5) that the parasites L. amazonensis spread in the liver of laboratory mice, although the parasites do not reproduce in these organs. 
the study of (16) indicated that the pathological effects of the liver are the most prominent with infiltration of inflammatory cells and the appearance of blood congestion and granuloma, and (3) noted the observation of hyperplasia of Koffer cells and infiltration of lymphocytes with enlarged liver cells, and (1) indicated that there are significant tissue changes in Collections of affected mice including hepatocyte enlargement, inflammatory cell accumulation, and lymphocyte infiltration.

Histological sections of the skin lesions visible on the ear, foot and tail regions showed the presence of the parasite in the dermis region, and this is consistent with what was mentioned by (11) that Leishmania parasites are not found inside the epidermis. Heavy infiltration of inflammatory cells such as lymphocytes and macrophage cells was also observed, and this is consistent with what was reported by (15) Abscesses as a result of the breakdown of inflammatory cells, especially neutrophils, due to their short life span compared to other cells, It is possible to explain why neutrophil cell debris produces antibiotic-like substances called definsins that release strong oxidizing substances that kill pathogens (2).

The results of the present study were in agreement with (20) of severe infiltration in the dermis layer mediated by neutrophil and polymorph lymphocytes, with the emergence of severe hemorrhage in the area of the dermis, with Necrosis in the epidermal cells penetrated by inflammatory cells.

And the increase in glutathione levels through NAC treatment improves the BALB/c mice response to infection as analysed using several critical parameters: histopathological outcome of the footpad lesion and cytokine profile of popliteal lymph node cells (14).

Conflict of Interest: The authors declare that they have no conflict of interest.

\section{Acknowledgements:}

We are very grateful for the by the University of Al-Qadisiyah. We also deeply thank the locals and persons who helped us during the continuous surveys that were conducted during the study period.

\section{References:}

[1] Al-Abbas, W. D. Sh. (2017). A comparative study to evaluate the effectiveness of Iraqi propolis extract and camel milk on the viability of the visceral parasite Leishmania donovani and its investigation in the sand fly vector (Diptera: Phlebotomus) using polymer chain reaction technique PCR. PhD thesis, College of Science, University of Kufa.

[2] Al-Abdullah, Sh. (2012). Physiology, first edition. House of the March for Publishing and Distribution, Amman, Jordan. The number of pages of the book is 534.

[3] Al-Khazraji, Z. A. H. (2005). Effect of lemongrass and Achillea extract on the parasite Leishmania tropica ex vivo and in vivo Master's thesis, College of Science, University of Baghdad: 116 pages.

[4] Akhoundi, M., Kuhls, K., Cannet, A., Votýpka, J., Marty, P., Delaunay, P. and Sereno, D. (2016). A historical overview of the classification, evolution, and dispersion of Leishmania parasites and sandflies. PLoS neglected tropical diseases 10(3). 
[5] Arfan, B. and Rahman, S. (2006). Correlation of clinical histopathological, and microbiological finding in 60 cases of cutaneous leishmaniasis. IJDVL., 72: 28-32.

[6] Bifeld, E. and Clos, J. (2015). The genetics of Leishmania virulence. Medical microbiology and immunology 204(6), 619-634.

[7] Blum, J., Lockwood, D.N., Visser, L., Harms, G., Bailey, M.S., Caumes, E., Clerinx, J., van Thiel, P.P., Morizot, G. and Hatz, C. (2012). Local or systemic treatment for New World cutaneous leishmaniasis? Re-evaluating the evidence for the risk of mucosal leishmaniasis. International health 4(3), 153-163.

[8] John D.T. and W.A. Petri .( 2006). Markell and Voge's Medical Parasitology.9th Ed. Saunders Elevier. New York, P:127-133.

[9] Karamian, M., Kuhls, K., Hemmati, M. and Ghatee, M.A. (2016). Phylogenetic structure of Leishmania tropica in the new endemic focus Birjand in East Iran in comparison to other Iranian endemic regions. Acta Tropica 158, 68-76.

[10] Kaye, P. and Scott, P. (2011). Leishmaniasis: complexity at the host-pathogen interface. Nature reviews microbiology 9(8), 604-615.

[11] Kurban, A.; Malak, J.; Farah, F. and Chaglassian, H.(1966). Histopathology of cutaneous leishmaniasis. Arch. Derm., 3: 396-401.

[12] Mcgwire, B.S. and Satoskar, A. (2014). Leishmaniasis: clinical syndromes and treatment. QJM: An International Journal of Medicine 107(1), 7-14.

[13] Postigo, J.A.R. (2010). Leishmaniasis in the world health organization eastern mediterranean region. International journal of antimicrobial agents 36, S62-S65.

[14] Rocha-Vieira, E., Ferreira, E., Vianna, P., De Faria, D. R., Gaze, S. T., Dutra, W. O., and Gollob, K. J. (2003). Histopathological outcome of Leishmania major-infected BALB/c mice is improved by oral treatment with $\mathrm{N}$-acetyl-1-cysteine. Immunology, 108(3), 401-408.

[15] Sangueza, O., Sangueza, J., Stiller, M. and and Sangueza, P.(1983). Mucocutaneous leishmaniasis: A clinicopathologic classification. J. Am. Acad. Dermatol., 28: 927-931.

[16] Santos Jamile Prado dos , Leucio Câmara Alves , Rafael Antonio Nascimento Ramos. and Aparecida da Gloria Faustino.(2013). Histological changes and immunolabeling ofLeishmania infantum in kidneys and urinary bladder of dogs. Rev. Bras. Parasitol. Vet. vol.22.

[17] Torres-Guerrero, E., Quintanilla-Cedillo, M. R., Ruiz-Esmenjaud, J., and Arenas, R. (2017). Leishmaniasis: a review. F1000Research, 6, 750.

[18] World Health Organization Regional Office for Africa, (2017). Leishmaniasis [WWW Document]. http://www.afro.who.int/health-topics/Leishmaniasis.

[19] World Health Organization WHO (2010). Factsheet338. Medicines: rational use of medicines. Geneva: WHO, Available at http://www.who. int/ mediacentre/ factsheets/ fs338/en. 
[20] Younes, N. N. (2018). Molecular and immunological diagnosis and experimental treatment of cutaneous leishmaniasis in mice and volunteer patients. $\mathrm{PhD}$ thesis, Institute of Genetic Engineering and Biotechnology, University of Baghdad. 